\title{
DE L'IMPACT DU RECENT RECRUTEMENT DES MAGISTRATS SUR LE SYSTEME JUDICIAIRE CONGOLAIS : UN PREMIER BILAN
}

\author{
Par Mundala Mundala Dieudonné ${ }^{1}$
}

\section{INTRODUCTION}

Dans leur mission de dire le droit, les cours et tribunaux civils et militaires ainsi que leurs parquets agissent au nom du peuple congolais et leurs décisions sont exécutées au nom du président de la république, chef de l'Etat ${ }^{2}$. Dans cet angle, l'appareil judiciaire du Congo a été renforcé pour une bonne administration de la justice par le recrutement d'environ deux mille nouveaux magistrats en vue de répondre efficacement aux préoccupations des justiciables.

Par ailleurs, les jeunes juristes, néophytes au sein de cet appareil judiciaire congolais déjà objet de plusieurs critiques parfois liées à la moralité douteuse de certains anciens magistrats, ont été qualifiés lors d'une allocution officielle du chef de l'Etat de « magistrats de la tolérance zéro ». Par la suite, le ministre de la justice de l' époque reprendra ces termes pour en faire un discours programme applicable au sein de ce troisième pouvoir régalien de l'Etat qui devrait subir une restructuration profonde. En effet, ces propos n'étaient que la conséquence directe du constat néfaste du dysfonctionnement de cet appareil judiciaire se caractérisant parfois par la corruption, le clientélisme etc. Par Ce discours, les autorités affichaient leur volonté de créer une démarcation entre la nouvelle génération.

Conscient de la grandeur de l'étendue de la république démocratique du Congo dont les cours et tribunaux n'étaient installés que principalement dans les centres urbains, il s'avérait impérieux de recruter des nouveaux magistrats non seulement pour couvrir tout le territoire par l'implantation des nouveaux tribunaux de paix(en principe un tribunal de paix pour chaque territoire) mais aussi en vue de suppléer à la carence en personnels judiciaires observée au sein des cours et tribunaux déjà fonctionnels.

Comme il fallait sélectionner des candidats valables, le recrutement a été précédé par l'adoption de la loi organique $\mathrm{n}^{\circ} 06 / 020$ du 10 octobre 2006 portant statut des magistrats. Et dans l'exposé des motifs de cette loi, parlant de l'indépendance des magistrats, il est renseigné ceci : « cette indépendance édictée dans toutes les constitutions que notre pays a connu jusqu'à ce jour, mais jamais suivies d'effets, doit, en cette période où la bonne gouvernance constitue le soubassement de toute action étatique, être comprise dans toutes ses implications conséquentes et traduites effectivement dans les actes.

1 Assistant à la Faculté de Droit de l'Université de Lubumbashi, mail: mundaladieudonne@yahoo.fr

2 Guide pratique de procédure disciplinaire des magistrats, USAID, Pro justice Médias Paul, Kinshasa, juillet 2011, p7. 
Dans cet ordre d'idées, il devient impératif que le pouvoir judiciaire, à la faveur du processus de démocratisation en cours, puisse réellement sortir du carcan dans lequel il a été confiné pour retrouver ses lettres de noblesse. Ainsi, ses animateurs que sont les magistrats pourront accomplir en toute indépendance, en toute conscience et en toute dignité, leur noble mission de rendre une bonne justice sans laquelle il n'y a pas de véritable paix civile dans la société, facteur indispensable à la stabilité politique ainsi qu'au développement économique et social $»^{3}$.

Autant dans cet exposé des motifs un accent particulier est mis sur l'indépendance des magistrats comme gage d'une bonne administration de la justice, autant les magistrats se doivent d'agir en toute conscience et dignité à cause de la noblesse de leur mission. C'est de la sorte que le récent recrutement réalisé en deux phases, d'abord le concours organisé à l'intention de tous ceux ayant déposé leurs dossiers auprès du bureau et ensuite ceux sélectionnés au premier tour qui devaient passer par une interview, a mis un accent sur la formation déontologique de futurs magistrats qui devraient, selon les initiateurs, être d'un atout positif et contribuer à l'amélioration du fonctionnement de la justice.

Trois ans après l'entrée en fonction de ces nouveaux magistrats, pouvons-nous affirmer que le changement tant attendu sur l'administration judiciaire est déjà arrivé? En guise de bilan de ce recrutement, est-il positif ou négatif? Autant de préoccupations auxquelles nous espérons pouvoir répondre.

Toutefois, vêtus des lunettes scientifiques et même celles d'avocat, régulièrement au prétoire et donc impliqué dans le système judiciaire congolais, nous serions in limine litis tentés de parler d'une simple illusion de sens. Car le changement n'est peut-être pas pour aujourd'hui. Bien au contraire, on se croirait dans la continuité plutôt que dans la rupture avec un passé triste quand il s'agit de l'aspect moral. Néanmoins, il semble souhaitable de nuancer notre point de vue du fait que l'augmentation de magistrats aura été à la base de l'ouverture de plusieurs tribunaux de paix dans les territoires jamais couverts par une juridiction. Bref, il s'agit d'un rapprochement de la justice des justiciables qui mérite d'être relevé parmi les points positifs.

Ainsi, la présente étude s'articulera autour de deux points : Le premier point relatif à l'impact de l'arrivée des nouveaux magistrats sur le fonctionnement de l'appareil judiciaire congolais montrera de quelle manière ce recrutement aura été une pierre apportée à l'édifice et dont l'importance reste indéniable en ce qu'il a contribué au renforcement du système judiciaire congolais. Le second point portera sur la critique des animateurs et analysera particulièrement l'apport des nouveaux magistrats au système.

3 Cfr Loi organique $\mathrm{n}^{\circ} 06 / 020$ du 10 octobre 2006 portant statut des magistrats. 


\section{DE L'IMPACT POSITIF DU RECRUTEMENT DES MAGISTRATS DE 2010 SUR LE FONCTIONNEMENT DE L'APPAREIL JUDICIAIRE CONGOLAIS}

Depuis l'accession de l'Etat du Congo à la souveraineté internationale comme Etat indépendant appelé à présider à ses propres destinées, au nombre des reproches formulés à son encontre figure notamment le dysfonctionnement de son système judiciaire. Pour un pays qui se veut un Etat de Droit, une bonne administration de la justice constitue le moteur par excellence de tout développement. En effet, on ne peut prétendre au développement économique et social tant que le système judiciaire ne tourne pas à plein régime. D'où l'intérêt particulier du chef de l'Etat de redresser ce troisième pouvoir en lui dotant des animateurs compétents.

Avant de vérifier l'apport du dernier recrutement des magistrats, disons un mot sur l'Etat lui-même comme entité devant mettre en place une bonne administration judiciaire et pour quelle fin?

Lorsqu'on cherche à comprendre l'Etat, on l'envisage comme une nation organisée administrée par un gouvernement. COMBACAU et SERGE SUR définissent l'Etat comme l'organisme chargé de la satisfaction de l'intérêt général, une autorité souveraine sur un peuple et un territoire déterminé. Une structure politique et administrative et dont se dote un groupement humain vivant sur un territoire donné et exerçant sur ce groupement et en son nom une autorité exclusive ${ }^{4}$.

L'Etat congolais compris ainsi comme cet organisme chargé de la satisfaction de l'intérêt général resterait comme une réalité abstraite sans parler du gouvernement. Pour que l'Etat existe juridiquement, il ne suffit pas d'être en présence d'un territoire et d'une population, mais il est nécessaire qu'il y ait un gouvernement effectif. En fait, l'effectivité du gouvernement s'entend comme la capacité du gouvernement à exercer, l'autorité politique ou le pouvoir sur l'ensemble du territoire, à le contrôler.

Parler de l'effectivité du gouvernement ramène également à parler de l'implantation des cours et tribunaux devant être effective sur toute l'étendue du pays. Si une grande partie du territoire surtout en milieu rural était non couverte par des juridictions, l'effectivité du système judiciaire serait absurde.

Ensuite, il est mieux de procéder par une approche sémantique du terme « magistrat » qui se trouve au centre de nos analyses et ce pour plus de compréhension du thème de notre réflexion.

Le terme Magistrat, pris dans le sens de la procédure pénale et civile, se définit conformément à la nature même de la juridiction au sein de laquelle il preste. Dans les juridictions de l'ordre judiciaire, les magistrats de carrière sont chargés de juger lorsqu'ils sont au siège, et de requérir l'application de la loi quand ils sont au parquet. Recrutés par concours ou par intégration sur titre, ils sont placés dans un statut distinct de celui des fonctionnaires ${ }^{5}$ et

4 COMBACAU et SUR, S., Droit international Public, PUF, 7ème éd. Paris, p21.

5 Ordonnance. $\mathrm{n}^{\circ} 58-1270,22$ déc. 1958. 
forment le corps judiciaire; ils jouissent, lorsqu'ils appartiennent au siège, de l'inamovibilité... Dans les tribunaux d'exception siègent des juges élus ou nommés, non professionnels qui ne sont pas des magistrats au sens exact du terme (juge du commerce, conseillers prud'homaux, juges de proximité, etc. ${ }^{6}$

De cette définition, il ressort que le terme magistrat est générique, car pouvant s'appliquer aux juges du siège comme aux officiers du ministère public chargés de requérir l'application de la loi au parquet.

Précisons également que ces jeunes magistrats n'ont eu que trois mois de stage au parquet avant de passer directement au siège(Tribunal) sans assez de maturité. En RDC, le magistrat du siège, appelé juge, a préséance sur celui du parquet.

\section{DU RAPPROCHEMENT DE LA JUSTICE DES JUSTICIABLES}

Parler d'une bonne administration de la justice revient à rendre celle-ci accessible aux bénéficiaires. Dans le souci de maintenir l'ordre public sur toute l'étendue du pays, il sied de rapprocher les institutions judiciaires des justiciables et ce par l'implantation des nouvelles juridictions.

Le gouvernement congolais pour être effectif devrait disposer véritablement dans les confins de tous les territoires, des juridictions afin d'éviter que dans certains coins la loi de talion ne puisse s'appliquer par manque de magistrats disponibles dans leur ressort. En effet, 10 ans après le recrutement de 1998, le système judiciaire se concentrait plus dans les grands centres urbains. L'effectif des magistrats du parquet comme ceux du siège étant insuffisant pour couvrir toute l'étendue.

C'est de la sorte que ce recrutement initialement annoncé de 2000 magistrats mais dont l'effectif total a été rabattu à 1972 a permis de résoudre quelques problèmes, à savoir:

1. La lenteur de l'administration judiciaire,

2. Le rapprochement de la justice.

\subsection{La lenteur de l'administration de la justice}

Pour les grandes villes dans lesquelles le nombre des contentieux se trouve croissant, par manque d'assez de magistrats surtout du siège, l'on a constaté avant le recrutement que plusieurs dossiers trainaient dans les tiroirs des juges qui ne savaient pas se prononcer dans le délai de la loi. Certains conflits s'allongeaient pendant des mois, voire des années pour qu'ils soient tranchés. Les statistiques d'avant renseignent que l'effectif total était de 1714 magistrats, soit 1 magistrat pour 30.000 congolais ${ }^{7}$. Alors qu'après le recrutement de 2009, l'effectif total des magistrats est passé à 3.686, soit 1 magistrat pour 16.277 habitants. Et ce

6 LEXIQUE DES TERMES JURIDIQUES, Dalloz, 17è édition, Paris, 2010, Ps 445 -446

7 KADIMA YVES, art.cit. p.2. 
conformément aux statistiques de l'OMS qui avance un effectif approximatif de 60.000 .000 de congolais en 2011 (mineurs et majeurs, hommes et femmes) alors que pour la commission électorale nationale indépendante, l'effectif total de la population adulte (en âge de voter) était de 32.024.640

En effet, l'augmentation du nombre des magistrats a permis un dénouement rapide de plusieurs conflits de tout genre (pénal, civil, foncier...). Cela figure parmi les acquis positifs de ce recrutement. Surtout pour les matières commerciales qui requièrent célérité, il était temps d'agir pour éviter toute la lourdeur administrative constatée au sein de l'appareil judiciaire congolais.

Comme le révèlent ces données chiffrées recueillies au Greffe Civil de le Cour d'Appel de Lubumbashi que nous plaçons dans un tableau :

\begin{tabular}{|c|c|c|}
\hline Période prise en compte & $\begin{array}{l}\text { Nombre des magistrats en } \\
\text { place. }\end{array}$ & $\begin{array}{l}\text { Nombres des dossiers pro- } \\
\text { noncés par les juges. }\end{array}$ \\
\hline \multicolumn{3}{|l|}{$\begin{array}{l}\text { Avant le recrutement du } 17 / \\
10 / 2009\end{array}$} \\
\hline $\begin{array}{l}\text { Période allant du } 01 \text { janvier au } \\
20 \text { décembre } 2009\end{array}$ & $\begin{array}{l}\text { La Cour d'appel de Lubumba- } \\
\text { shi ne comptait que } 7 \text { juges y } \\
\text { compris son premier Président. }\end{array}$ & $\begin{array}{l}153 \text { dossiers prononcés, y com- } \\
\text { pris les Avant Dire Droit et les } \\
\text { Défenses à exécuter. }\end{array}$ \\
\hline \multicolumn{3}{|l|}{ Après le Recrutement } \\
\hline $\begin{array}{l}\text { Période allant du 20/06/2013au } \\
30 / 12 / 2013\end{array}$ & $\begin{array}{l}\text { La Cour d'appel de Lubumba- } \\
\text { shi compte actuellement } 12 \\
\text { juges, y compris le } 1^{\text {er }} \text { pré- } \\
\text { sident }\end{array}$ & $\begin{array}{l}156 \text { dossiers prononcés y com- } \\
\text { pris les Défenses à exécuter et } \\
\text { les Avant dire Droit. }\end{array}$ \\
\hline $\begin{array}{l}\text { Période allant du 01/01/2014 à } \\
\text { nos jours }\end{array}$ & 12 juges & $\begin{array}{l}53 \text { dossiers prononcés avec ar- } \\
\text { rêts définitifs sans compter les } \\
\text { Défenses à exécuter et les } \\
\text { Avant Dire Droit }\end{array}$ \\
\hline
\end{tabular}

9

Pour cela, la République Démocratique du Congo a bénéficié de l'appui de plusieurs partenaires dont la majorité est constituée des ONG étrangères. Tel est le cas du volet appui à la justice qui est l'un des volets de la gouvernance judiciaire et sécuritaire. Sa principale vocation est d'appuyer la mise en œuvre du plan d'action pour la réforme de la justice en République Démocratique du Congo, à travers plusieurs axes d'intervention à savoir : accès à la justice, la lutte contre la corruption, et les violences sexuelles, appui institutionnel (qui

8 "Echos des Elections", in Magasine d'informations Electorales en République Démocratique du Congo, numéro spécial, $\mathrm{n}^{\circ} 001$, septembre 2011, p.9 L'administration étant défaillante du fait que le dernier recensement date de 1984, nous nous sommes fiés aux données de l'OMS.

9 Ces données ont été obtenues au Greffe Civil le 27 mars 2014 et sont disponibles dans le Registre Civil de La Cour d'appel de Lubumbashi. 
vise les hautes cours de justice), appui au personnel judiciaire, appui à la reforme pénitentiaire ${ }^{10}$.

Cette ONG dénommée :" Appui à la justice", a démarré ses activités en RDC le 01/ décembre 2009 avec un budget d'USD 1801.223,16. Les principaux donateurs étaient le PNUD et la France. Parmi les résultats atteints dans le cadre d'une bonne administration de la justice, il y a entre autres :

- Une campagne de sensibilisation et vulgarisation sur l'assistance judiciaire gratuite réalisée à Kinshasa à l'intention des personnes vulnérables;

- Appui fourni à l'organisation de la deuxième phase de la formation initiale à l'intention des 1000 magistrats nouvellement recrutés;

- Organisation des ateliers sur la carrière et la discipline à l'intention de nouveaux magistrats provinciaux; renforcement des capacités techniques et professionnelles des magistrats sur le traitement du contentieux électoral à Kinshasa, Lubumbashi et Matadi...

Parmi les principales activités menées dans ce domaine afin de redynamiser ce système judiciaire congolais, l'on peut citer entre autres : - le soutien à la commission permanente de réforme du droit congolais en sigle CPRD et la commission ministérielle dans la finalisation et la validation du projet de loi sur l'assistance judiciaire gratuite en RDC;

- le soutien du conseil supérieur de la magistrature(CSM) dans le recrutement et la formation de 2000 magistrats, dans l'organisation de la carrière et la discipline des magistrats dans six provinces notamment par le fonctionnement de six chambres provinciales de discipline et la formation sur le traitement du contentieux électoral ${ }^{11}$.

En clair et comme démontre KADIMA YVES, dans le cadre de la restauration d'un Etat de Droit et d'une magistrature indépendante, le gouvernement de la RDC, a décidé d'organiser un concours pour toute personne qui désire devenir magistrat. Test qui a eu lieu du 17 au $20 / 10 / 2009 »^{12}$

A cet effet, un test oral a été organisé afin de sélectionner les deux mille candidats. Et par la décision $\mathrm{n}^{\circ} 001 / \mathrm{CSM} / \mathrm{SPCSM} / \mathrm{D} / \mathrm{J} / 2010$ du 23 mars 2010, mille magistrats ont été retenus pour l'année 2010 et enfin par la décision n002/CSM/SPCSM/D/J/2010 du 23 mars, mille autres magistrats ont été réservés pour 2011.

Le programme de recrutement des magistrats visait à combler le fossé observé dans le domaine judiciaire et qui occasionnait un déficit dans l'administration de la justice sur le plan aussi bien quantitatif que qualitatif. Il se veut également une réponse à l'impératif de la trilogie exécutif - législatif - judiciaire ${ }^{13}$.

10 www.cd.undp.org, consulté le 13 janvier 2013, 12h 33'.

11 Les 6 provinces concernées sont le Bas Congo, le Kasaï-Oriental, le Kasaï-Occidental, le Nord Kivu, La Province Orientale et l'Equateur.

12 1. KADIMA YVES, « Recrutement de nouveaux magistrats », in www. Net, article du 02 avril 2011, p.1, Consulté le 10 janvier 2013 à 11h26'.

13 IDEM, P2. 


\subsection{Du rapprochement de la justice des justiciables.}

L'impact du recrutement de 1972 nouveaux magistrats a augmenté ipso facto leur nombre qui est passé de 1714, soit 1 magistrat pour 30.000habitants, à 3686 magistrats pour une population qui avoisine actuellement 60 millions répandus sur toute l'étendue du Pays. Du coup, plusieurs parquets secondaires ont été installés et par la suite l'implantation de tribunaux de paix dans plusieurs territoires de l'arrière-pays est devenue réalité.

C'est dans cette lancée que pour le ressort de la Province du Katanga, au regard de l'organigramme dressé par le Greffier Principal, 14 nouveaux Tribunaux de Paix ont été implantés ${ }^{14}$. Ce qui porte le nombre total à 29 Tribunaux de Paix opérationnels. L'effectif de Tribunaux de Paix pour le Katanga n'était que de 15 avant l'arrivée de nouveaux magistrats. Toujours dans la même logique, 16 nouveaux parquets près les Tribunaux de Paix ont été rendus opérationnels pour le ressort du Katanga et leur effectif total est passé de 11 à $27^{15}$.

Pour des habitants des territoires de Kabalo, Nyunzu, Moba par exemple, cela était comme un soulagement, car ils n'étaient plus obligésd'effectuer des centaines des kilomètres afin de rencontrer un magistrat à Kalemie. Ce rapprochement profite plus à ceux qui habitent les coins reculés des provinces.

Par ailleurs, l'implantation des Tribunaux de Paix ainsi que des Parquets secondaires dans des territoires jadis gérés par les administrateurs des territoires et par les Chefs coutumiers a eu une influence sur ces derniers qui se sont vus refuser la prérogative de continuer à juger certaines affaires. En fait, le Tribunal de Paix remplace les anciens Tribunaux de Police et ceux coutumiers. Lorsqu'on installe un Tribunal de Paix, le Tribunal coutumier disparaît et même celui de Police. Et en revanche, ces autorités coutumières n'ont pas réservé un accueil chaleureux aux magistrats venant d'ailleurs.

L'illustration est faite en évoquant la difficulté d'installation rencontrée par le tout nouveau Président du Tribunal de Paix Kongolo qui s'est heurté à une résistance des médecins locaux de Kongolo ne voulant pas lâcher l'immeuble alors qu'il était inhabité. L'administrateur de Territoire avait préféré prendre fait et cause pour les médecins pendant que le président de la juridiction logeait encore dans un hôtel de la place. Il faut rappeler qu'avant l'arrivée du Tribunal de Paix, il existait un Tribunal de Police et coutumier qui était présidé par l'administrateur assisté par certains chefs de Tribus. Et donc l'installation du tout nouveau Tribunal de Paix n'était pas forcément bien perçue par tous ${ }^{16}$.

1414 nouveaux Tribunaux de Paix pour le Katanga : Kamina, Kabongo, Kanyama, Kalemie, Kabalo, Moba, Nyunzu, Mitwaba, Kipushi, Kasenga, Sandowa, Kapanga, Dilolo et Mutshatsha.

1516 nouveaux parquets près les Tribunaux de Paix du Katanga : Mutshatsha, Kapanga, Sandowa, Dilolo, Moba, Kalemie, Kabongo, Kanyama, Kamina, Kolwezi, Likasi, Kasenga, Kipushi, Lubumbashi/ Katuba, Lubumbashi/ Kamalondo et Lubumbashi/Rwashi.

16 La Radio Okapi a fait échos de ce conflit se traduisant par l'arrestation d'un Médecin local ayant entrainé une grève de tous les médecins de l’Hôpital Général de Kongolo, grève de deux jours en juin 2011. 
Le pouvoir constituant du 18 février 2006 comme législateur de la loi organique $\mathrm{n}^{\circ} 06 / 020 \mathrm{du} 10$ octobre 2006 portant statut des magistrats et celui de la loi organique $\mathrm{n}^{\circ} 08 / 013$ du 05 Août 2008 portant organisation et fonctionnement du conseil supérieur de la magistrature ont adhéré à ce postulat élémentaire de bonne gestion. C'est pourquoi, autant ils n'ont ménagé aucun effort pour renforcer l'indépendance du pouvoir judiciaire de manière à la rendre effective, autant, au fil du temps, ils ont concouru à préciser les concours d'un régime disciplinaire des magistrats qui soit adapté aux exigences des Etats démocratiques modernes ${ }^{17}$.

Par ce renforcement de la procédure disciplinaire des magistrats, les justiciables trouvent des garanties de bonne administration de la justice et se rapprochent un peu plus de la bonne justice et équitable qui veut que chacun réponde de ses actes sans tenir compte de son statut de magistrat. Certes les magistrats bénéficient des privilèges de juridiction mais en définitive ils doivent répondre de leurs propres actes. Par exemple, un juge qui sait que s'il est surpris en train de prendre un verre de bière avec l'une des parties au procès, il peut être récusé par l'autre partie, il évitera tout comportement pouvant le compromettre.

Tout en parlant du rapprochement de la justice des justiciables, signalons également qu'en rapport avec l'indépendance des magistrats, la révision constitutionnelle de 2011 qui a porté sur environ 8 articles dont l'article 149 de cette même constitution, place dorénavant les magistrats du parquet sous l'autorité hiérarchique du ministre de la justice. C'est dans cette optique que l'indépendance dont question supra ne s'applique qu'aux seuls magistrats assis du siège appelés communément « juges ». Et pourtant, au regard de la constitution du 18 février 2006, l'indépendance du pouvoir judiciaire figurait dans le bloc dur, comprenant les matières qui ne doivent pas faire l'objet d'une révision constitutionnelle.

\section{ANALYSE CRITIQUE DE L'APPAREIL JUDICIAIRE CONGOLAIS ACTUEL}

Quasiment trois ans après la prise des fonctions de cette nouvelle promotion des magistrats, pouvons-nous affirmer que la visée des initiateurs a été atteinte? Si précédemment nous avons essayé de démontrer que cela a marché, cependant il est impérieux de relativiser les acquis pour mieux cerner les faiblesses. Que ce soit à l'étape du recrutement comme à celle de l'expérimentation, des critiques peuvent être formulées en vue de redynamiser de plus en plus cet appareil judiciaire que tout le monde (politique comme scientifique ou commun de mortel) veut voir assumer totalement son rôle.

\section{II.1. CRITIQUE DE LA PHASE DE RECRUTEMENT DES MAGISTRATS.}

Conformément au prescrit de l'article $1^{\text {er }}$ de la loi organique nº6/020 du 10 octobre 2006 portant statut des magistrats, plusieurs conditions devraient être réunies par les impétrants :

17 USAID/ Pro justice, op. cit., p11. 
1. Posséder la nationalité congolaise;

2. Être âgé d'au moins vingt et un ans accomplis et n'avoir pas dépassé l'âge de quarante ans;

3. Jouir d'une parfaite moralité attestée par un certificat délivré par une autorité administrative et par un extrait de casier judiciaire;

4. Jouir de la plénitude de ses droits civiques;

5. Posséder les aptitudes physiques et mentales attestées par un certificat médical daté d'au moins trois mois au dépôt du dossier au secrétariat permanent du conseil supérieur de la magistrature;

6. Être titulaire d'un diplôme de docteur ou de licencié en droit délivré par une université nationale publique ou privée légalement agréée ou d'un diplôme délivré par une université étrangère déclarée équivalent conformément à la législation congolaise sur l'équivalence des diplômes;

7. S'il s'agit d'une personne mariée, produire un extrait d'acte de mariage ${ }^{18}$.

Toutes les 6 premières conditions sont cumulatives à l'exception de la septième qui ne s'applique qu'aux impétrants mariés. Juridiquement le fait que l'une manque devrait entraîner le rejet du dossier du candidat. Sur les treize mille dossiers enregistrés au secrétariat, 6700 seulement furent déclarés valables et recevables par une commission mise sur pied par le conseil supérieur de la magistrature.

De ces six mille sept cents candidats, après le concours sus-évoqué, 2500 seulement seront retenus. Et compte tenu des moyens financiers limités pour le paiement de ces nouveaux magistrats, le gouvernement de la République Démocratique du Congo a par le truchement du ministre de la justice demandé au Conseil Supérieur de la Magistrature de ne retenir que deux mille.

\section{II.1.1. Manque d'objectivité dans le recrutement des nouveaux magistrats.}

Alors que la politique du gouvernement, agissant par le biais du ministre de la justice, consistait à tout prix à rompre avec les mauvaises pratiques qui avaient déjà élu domicile dans cette institution étatique, une liste non numérotée, scellée et signée conjointement par le Premier Président de la Cour Suprême de Justice, en sa qualité de Président du Conseil Supérieur de la Magistrature et, par le Secrétaire Permanent dudit Conseil, a été affichée aux sièges de toutes les Cours d'appel de Kinshasa/Gombe et de Kinshasa/ Matete, publiée sur le site internet du ministère national de la justice.

Contre toute attente, alors que les candidats étaient dans l'attente pour le début de la formation, une nouvelle liste numérotée et signée cette fois-ci par le seul Secrétaire Permanent du Conseil Supérieur de la Magistrature et sur laquelle certains noms ne figuraient

18 Loi organique $\mathrm{n}^{\circ} 06 / 020$ du 10 octobre 2006 portant statut des magistrats, in Journal officiel du 25 octobre 2006.

KADIMA YVES, art.cit. p2. 
plus, sera dressée le 29 janvier 2011 et affichée seulement devant le bâtiment de la Cour Suprême de Justice. Les candidats victimes de cette erreur administrative ou de cette omission réfléchie l'ont dénoncée à travers une correspondance adressée non seulement au Président de la République mais aussi au Président du Sénat ainsi qu'aux partenaires ayant permis l'organisation du test ${ }^{19}$.

Pour ces impétrants victimes de cette situation, cette omission qui avait l'impression d'être involontaire, cédait place à un comportement délibéré au profit des candidats dont les noms ne figuraient pas sur la liste officielle( $\left.1^{\text {ère liste }}\right)$, cela à cause du silence qui régnait au niveau du ministère de la justice relativement au sort leur réservé. Cela étant, ils sollicitaient l'interpellation du ministre national de la justice à la chambre basse du parlement ainsi que de tous ceux qui étaient impliqués dans le dossier afin d'aboutir à une solution qui permettrait de témoigner qu'effectivement la rigueur dont question quand on qualifie ces magistrats de " tolérance zéro" et de l'instauration d'un véritable Etat de droit au Congo était lancée ${ }^{20}$.

La rigueur tant souhaitée commençait à disparaître pour devenir un simple slogan. Car les ennemis du changement, ont profité de ce flou pour changer plusieurs noms de candidats ayant déjà satisfait en les remplaçant par les leurs ne réunissant pas parfois les critères prédéfinis à l'article premier de la loi précitée. La réussite à ce concours devrait avoir pour assise une très grande objectivité dans la sélection des candidats futurs magistrats. Cette confusion des listes n'a fait qu'attiser le feu de la subjectivité.

Ce concours n'était plus objectif du fait que certains candidats étaient passés dans des conditions floues. Ces derniers que les autorités politiques souhaitaient qu'ils s'inscrivent dans la logique de rigueur professionnelle, ont opté pour la continuité. L'espoir d'apporter un changement devenait mince pour un candidat recruté dans ces conditions peu objectives. Le virement à quatre-vingt-dix degrés des mentalités par rapport aux ainés dans la magistrature ne devenait qu'une simple utopie.

Par ailleurs, tous ces candidats victimes de magouilles orchestrées au niveau du secrétariat du conseil supérieur de la magistrature, n'ont jamais été entendues ni rétablies dans leurs droits. Sur ce point, il n'y a pas eu de suivi de la part du ministère national de la justice qui avait tout intérêt à assainir totalement ce corps. Le ministre de la justice lui-même n'a jamais été interpellé au parlement quant à ce.

De ce qui précède, nous pouvons affirmer sans peur d'être contredit que le recrutement des nouveaux magistrats n'a pas été objectif, le système de parrainage s'est taillé une place à travers les listes contradictoires qui étaient affichées. Ceux des candidats qui avaient un proche dans cette commission savaient sur qui s'appuyer pour faire figurer leurs noms sur la liste de sélection.

A titre illustratif, il nous semble souhaitable de présenter l'un des tableaux trouvés sur le site de la radio Okapi et qui démontre à suffisance que plusieurs conditions exigées à

19 KADIMA YVES, art.cit. p2.

20 Ibidem. 
l'article premier de la loi organique portant statut des magistrats ${ }^{21}$ ont été violées. Ce tableau révèle que certains candidats n'auraient pas pu être retenus du fait qu'ils ne remplissaient pas les conditions requises.

21 www.cd.undp.org, Tableau consulté 12 janvier 2013 à 12h 12'. 


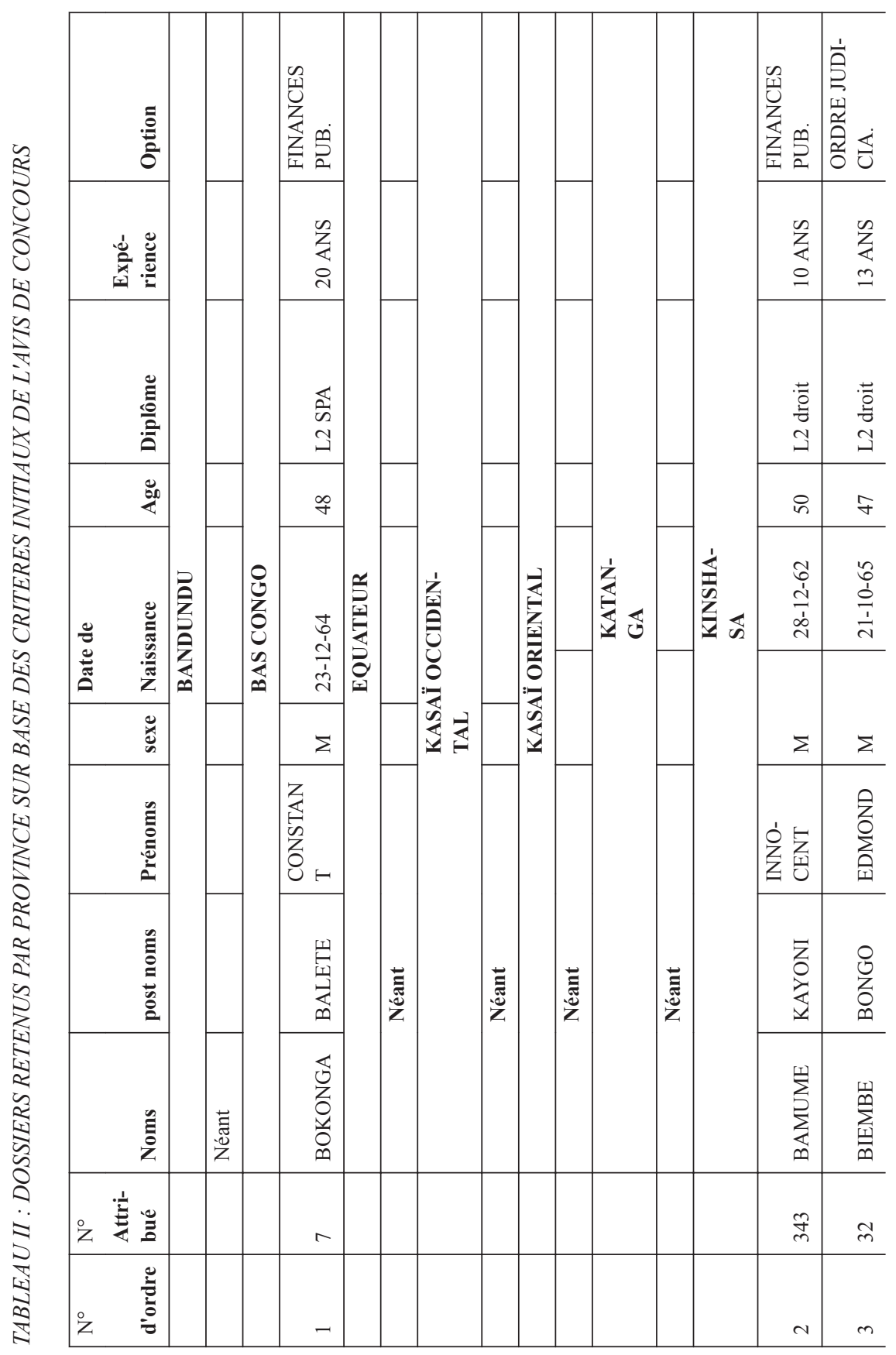




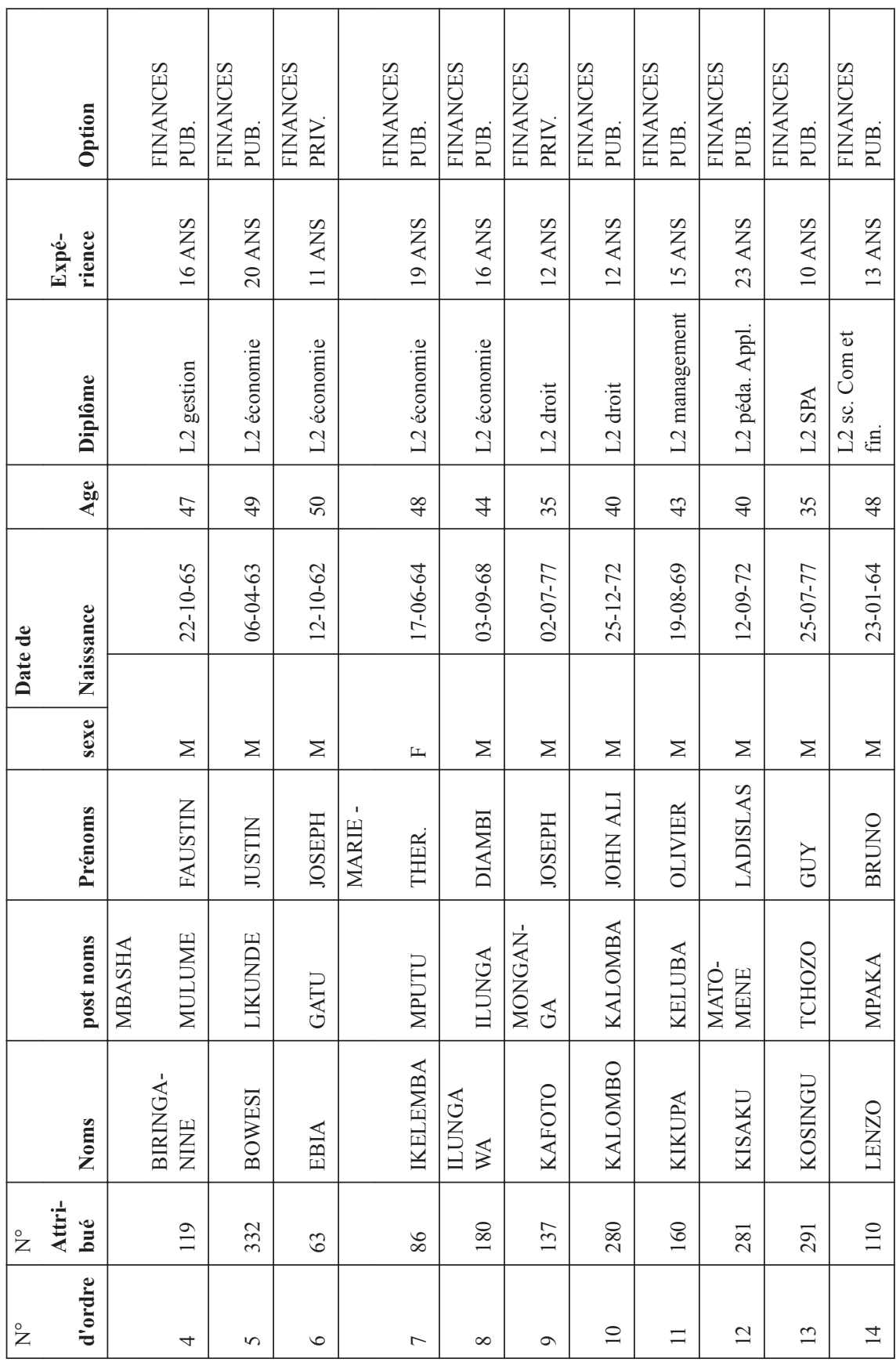


DE L'IMPACT DU RECENT RECRUTEMENT DES MAGISTRATS SUR LE SYSTEME JUDICIAIRE CONGOLAIS : UN PREMIER BILAN

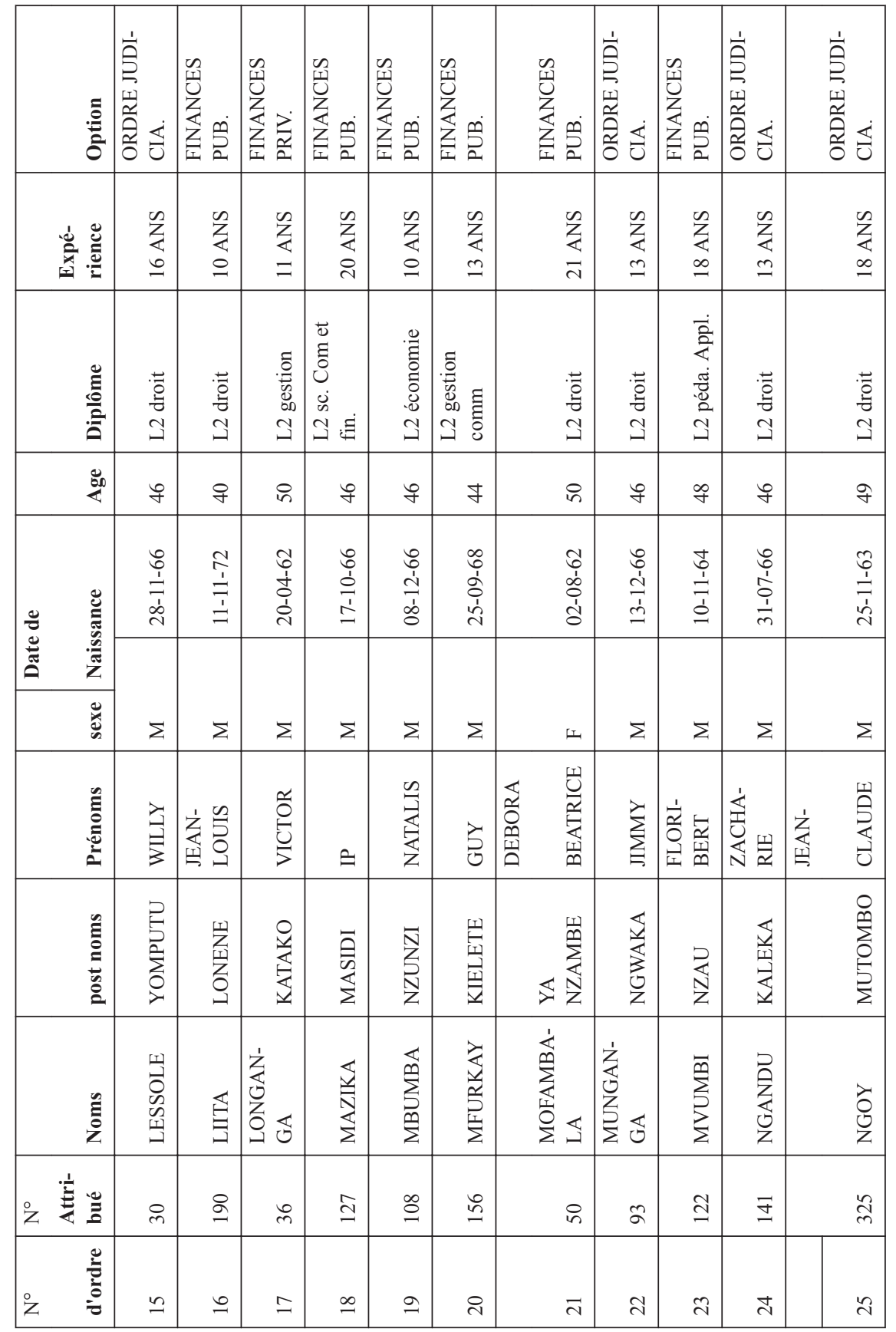




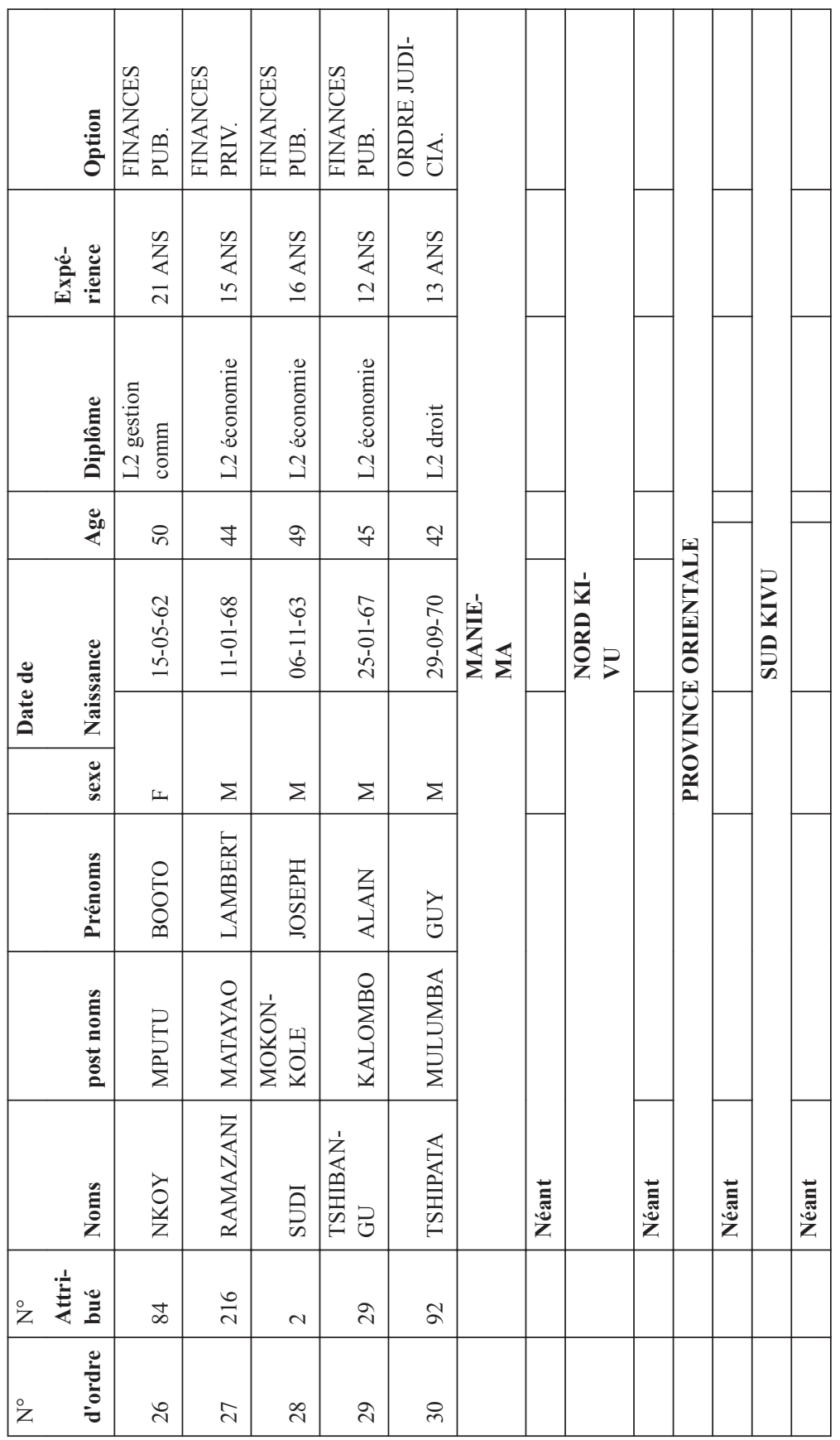


II.1.2. Du non-respect de la 6ème condition de l'articeler précité dans la phase de recrutement.

Au regard de plusieurs tableaux recueillis sur le site internet de la Radio Okapi, plus précisément le tableau $\mathrm{n}^{\circ} 2$ sus évoqué, il ressort que ce recrutement a été entaché de plusieurs irrégularités. En effet, la $6^{\text {ème }}$ condition énumérée à l'article premier de la loi précitée a été manifestement violée. Il semble souhaitable de préciser qu'en dépit du fait que ce tableau trouvé sur le net ne devrait pas être considéré comme une vérité absolue, il reflète néanmoins,à notre humble avis, une vérité relative, déjà objet de quelques rumeurs mais que nous attendions confirmer par des traces comme ce tableau qui reprend des candidats magistrats n'ayant pas un diplôme de droit.

Ce tableau renseigne que sur 30 dossiers retenus par province sur base des critères initiaux de l'avis de concours, uniquement 11 impétrants étaient porteurs d'un diplôme de licencié en droit, 8 détenaient celui de licencié en économie, 4 ont déposé un diplôme de licencié en gestion, 2 en pédagogie appliquée, 2 autres celui de licencié en sciences politicoadministrative, encore 2 diplômes de licencié en sciences commerciales et financières et enfin un diplôme de licencié en management. Il ressort que 19 candidats ne remplissaient pas cette $6^{\text {ème }}$ condition qui paraît, à nos yeux, capitale même pour accéder à la carrière de magistrats. Ce tableau reprend même les identités des candidats concernés au point où il paraît digne de foi.

En outre, rien ne permet d'affirmer que les 11 licenciés en droit provenaient des universités officielles ou privées légalement agréées.

1. Du non-respect de la deuxième condition du même article précité.

A la lumière de l'article premier précité, le candidat au concours de la magistrature devrait être âgé d'au moins 21 ans accomplis et n'avoir pas dépassé l'âge de quarante ans. Au regard du tableau2 sus évoqué, il appert que le critère âge a été manifestement violé.

Le point de départ reste la date du 17 au $20 / 10 / 2009$ au cours de laquelle le conseil supérieur de la magistrature a organisé le dit concours. Les candidats les moins âgés pouvaient, conformément à l'alinéa2, être ceux dont la date de naissance était l'an 1988 et les plus âgés ne devraient pas être nés avant octobre 1969. Tous ceux qui ont vu le jour avant cette année 1969, totalisaient plus de quarante ans au moment du dépôt des dossiers.

De ce qui précède, l'on peut noter que 23 des dossiers retenus n'auraient pas dûs l'être pour raison d'âge avancé. Mais curieusement ce sont ceux qui sont censés faire respecter la loi qui se permettent de la fouler au pied. Pourquoi produire des textes de lois que l'on ne sait pas mettre en application. L'on a admis même ceux qui avaient dépassé 45 lors du test. Eu égard à la loi organique ${ }^{\circ} 06 / 020$ du 10/10/2006, toutes ces irrégularités observées devraient avoir comme conséquence la révocation des magistrats nommés sans qu'ils aient préalablement remplis les conditions prévues à son article premier. 
En sus, un autre détail qui semble négligeable, c'est que 29 dossiers sur 30 retenus dans ce tableau provenaient des candidats de la province-ville de Kinshasa, capitale du Congo et siège national de la majorité des institutions. L'on comprendrait facilement que les interventions ne sont en fait que l'œuvre des différents responsables politiques. Voilà une illustration des interférences politiques et même une violation du principe de l'indépendance de la magistrature.

Car une situation que l'on peut observer et qui n'est plus un secret de polichinelle, c'est que lorsque le ministre de la justice est originaire de telle province, les ressortissants de sa province se retrouveront plus et coifferont même plusieurs juridictions pendant son mandat.

Enfin, lorsqu'on annonce le recrutement des nouveaux magistrats, les responsables ont précisé le besoin de nommer 2000 magistrats, à raison de mille directement et mille autres qui suivraient dans l'année suivante. Curieusement, le chef de l'Etat a nommé mille d'abord et puis 972. Où est passé le budget de28 autres magistrats qui manquaient sur cette deuxième liste. A qui profite ce décalage des chiffres et que faire pour atteindre le nombre arrêté par le gouvernement et ses partenaires? Pourquoi recruter1972 magistrats au lieu de 2000 déjà annoncés officiellement? A l'heure actuelle, personne ne peut affirmer que le nombre des magistrats suffit pour tout le Pays, il est impérieux d'en ajouter encore dans le futur.

Autour du recrutement, les critiques les plus acerbes peuvent à foisonner, car la réussite de ce recrutement dépendrait entièrement de l'objectivité de cette opération.

\section{II.2. CRITIQUE DE LA PHASE D'ENTREE EN FONCTION DES NOUVEAUX MAGISTRATS}

Dans cette phase, il est question de voir le rendement des magistrats de "la tolérance zéro" sur l'appareil judiciaire. Nous aurons un intérêt pour d'abord ceux qui sont passés presque directement au siège et enfin nous terminerons avec ceux du parquet. C'est ici qu'il convient de dresser un premier bilan de la présence de ces nouveaux magistrats dans l'appareil judiciaire. L'exercice va consister à ressortir les faiblesses constatées depuis l'entrée de cette dernière génération des magistrats dans le corps judiciaire.

\section{II.2.1. Rendement des nouveaux magistrats du siège sur l'administration de la justice.}

Les magistrats dit" de la tolérance zéro" qui sont au siège, accusent beaucoup de lacunes pour l'instruction des dossiers aux audiences et ont des difficultés réelles pour se prononcer sur la saisine du tribunal à l'égard des parties. Les habitués du prétoire (Avocats) en savent plus. Le passage au parquet aurait dû se faire sous la forme d'un stage relativement long. Par le passé, tout magistrat nouvellement recruté devait passer assez de temps au parquet pour mieux être rodé avant de passer au siège. Cette pratique garde toute son importance et se justifie encore aujourd'hui. La théorie apprise à l'université se décale un peu de la pra- 
tique et il est mieux que le futur magistrat commence par le parquet pour acquérir plus d'expérience nécessaire pour l'instruction des dossiers.

Comment est -ce possible que le passage du parquet vers le siège, considéré comme une promotion, ait pu bénéficier aux nouveaux magistrats qui n'avaient que trois mois de stage au parquet, sans expériences suffisantes sur le système judiciaire congolais? Techniquement les membres du conseil supérieur de la magistrature devaient comprendre que cela n'irait pas sans poser un problème sérieux.

Par ailleurs, du point de vue déontologique, les nouveaux magistrats ne paraissent pas s'inscrire dans la rupture avec leurs prédécesseurs. Le souci d'une bonne administration de la justice est relégué au second plan et la priorité est de vite s'enrichir. Ils sont de fois plus que la copie servile des aînés déjà diabolisés. Un jeune magistrat qui touche un salaire moyen avoisinant $500 \$$ américains mettrait suffisamment des temps pour s'acheter une voiture de luxe. Si l'on observe certains qui s'enrichissent en un laps de temps, l'on peut présumer qu'ils aient plongé dans des pratiques tant décriées.

La paix civile qui est le facteur indispensable à la stabilité politique et au développement économique et social ne peut être que le fruit d'une bonne administration de la justice. Le nouveau juge ne devrait pas faire trainer aux tiroirs certains dossiers alors qu'ils sont en état de recevoir les prononcés. Voilà ce qui était reproché au juge d'avant la tolérance zéro et qui se constate encore aujourd'hui chez certains.

II.2.2. Etat actuel du parquet après l'entrée en fonction des magistrats de la tolérance zéro.

Parmi les grandes critiques liées au comportement professionnel formulées à l'encontre des magistrats du parquet avant et après l'arrivée de la dernière génération, figurent entre autre les arrestations et détentions préventives injustifiées. L'article 27 du code congolais de procédure pénale énumère les conditions qui peuvent autoriser un officier du ministère public à placer un inculpé en détention préventive. Il ressort de cette disposition que l'inculpé peut être placé en détention préventive lorsque sa fuite est à craindre, son identité est douteuse ou inconnue et qu'il existe contre lui des indices sérieux de culpabilité...

Le pouvoir de placer sous mandat d'arrêt provisoire n'a été accordé à l'officier du ministère public que pour répondre aux besoins d'action immédiate. Antoine RUBBENS estime que le juge n'a pas la qualité d'apprécier la légalité de la détention antérieure à son intervention, sa mission consiste uniquement à vérifier si à la date de son audience en chambre du conseil, les conditions justifiant la mise en état de détention préventive sont réunies telles que prévoit l'article 28 du code de procédure pénale 22 .

En pratique, même après l'entrée en fonction de ces magistrats dits de «tolérance zéro », l'on observe plusieurs abus de pouvoirs qui n'ont pour visée que la recherche du gain illicite. L'unique moyen de se faire l'argent rapidement est de placer un inculpé en état d'ar-

22 RUBBENS, A., Le droit judiciaire congolais, l'instruction criminelle et procédure pénale, T2, MsonF., Larcier,, S.A., Bruxelles, 1965. 
restation afin qu'il verse une caution et que la liberté provisoire lui soit accordée. Pas de souci pour une bonne administration de la justice qui amènerait l'organe de la loi à commencer par vérifier si les conditions de l'article 27 sont réunies ou pas. Surtout lorsqu'il s'agit de ressortissants étrangers qui passent pour une proie facile à intimider afin de s'enrichir rapidement sans cause. Comme dit précédemment, rien ne permet de dire qu'il y a eu rupture. Bien au contraire, cette dernière promotion s'est placée dans la continuité, voire même dans le dépassement.

Dans la même logique, certains nouveaux magistrats affectés dans des Tribunaux de paix nouvellement installés se retrouvent parfois obligés d'assumer l'intérim du Procureur de la république alors qu'ils n'ont même pas bénéficié d'un encadrement suffisant pour des pareilles responsabilités. Signalons que sur initiative des ONG de droits de l'homme, certaines formations de renforcement des capacités des magistrats sont tenues à l'intention de ces nouveaux magistrats.

Déontologiquement cette génération des magistrats est également mal en point. Ils sont dans les débits de boissons comme leurs aînés pour boire et s'exhiber un pas de danse alors que la noblesse de leur carrière. Dernièrement un incident bizarre a conduit l'un des nouveaux magistrats en poste au Parquet de Kasumbalesa devant la cour d'appel de Lubumbashi pour y répondre de ses actes. Au premier degré, il a même été condamné à une peine de six mois de servitude pénale mais comme l'affaire est en cours, étant donné qu'il a interjeté appel devant la cour suprême de justice, nous avons l'obligation de réserve en gardant l'anonymat d'autant plus que le dossier n'a pas encore connu une décision définitive.

Le premier bilan de l'arrivée des nouveaux magistrats est mitigé, car l'appareil judiciaire congolais n'est pas dans l'état souhaité et la révolution des mentalités tant attendue n'est pas pour aujourd'hui. Parfois les correcteurs se sont avérés pires que ceux qu'il fallait amender. Au début ils ont été accueillis dans un climat de méfiance, car les anciens attendaient voir le changement qu'ils allaient entraîner. Ayant compris qu'ils s'inscrivaient dans la continuité, ils ont formé un bloc commun pour garder le cap.

\section{CONCLUSION}

En guise de conclusion, l'impact des récents recrutements des magistrats sur les aspects tant organisationnel que structurel du système judiciaire congolais présente un bilan vraiment mitigé. Il est vrai que les commentaires faits par le ministre de la justice, et surtout le qualificatif de " magistrats de la tolérance zéro » ont poussé les justiciables à nourrir beaucoup d'attentes. Mais la déception aura été à la hauteur de l'attente même s'il est indéniable que les points positifs n'ont pas manqué.

L'augmentation du nombre des magistrats a eu néanmoins des répercussions positives. Elle a, dans la foulée, favorisé l'implantation de plusieurs Parquets secondaires ainsi que des Tribunaux de paix dans plusieurs territoires non couverts jusque-là par le système judiciaire congolais. De la sorte, ce recrutement a favorisé le rapprochement de la justice des 
justiciables qui n'ont plus à effectuer des longues distances afin de trouver le dénouement à leur litiges.

Toujours dans cette même lancée, il appert sans doute que l'augmentation du nombre des magistrats a quand même un effet sur la rapidité dans le prononcer des décisions judiciaires. Les justiciables n'ont plus à attendre très longtemps comme avant pour recevoir un jugement. Cependant, il convient de nuancer cette déclaration du fait que les juges véreux continuent toujours à faire traîner les dossiers dans leurs tiroirs, le temps de se faire corrompre par les parties. Pour ce cas, il suffit que l'avocat conseil écrive au président de la juridiction pour que le juge fautif soit interpellé et même poursuivi disciplinairement.

Cependant, l'arrivée de cette dernière génération des magistrats n'a pas été que rose, car elle équivaudrait parfois à une montagne qui n'a accouché que d'une sourie.

Nous avons longuement tablé sur la qualité du recrutement qui a perdu de son objectivité à cause des multiples interférences des politiques sur les membres du conseil supérieur de la magistrature qui ont fini par tomber dans le piège qu'il fallait à tout prix éviter. A partir du moment où ce recrutement a été raté, en admettant des candidats qui ne remplissaient pas les conditions énumérées à l'article premier susmentionné, il devenait superfétatoire d'attendre d'eux un travail de qualité. La qualité aurait dû se faire sentir dans la sélection.

De la sorte, il s'est avéré normal que les candidats recrutés dans les conditions décrites précédemment, ne pouvaient s'inscrire que dans la logique de la continuité. Les nouveaux magistrats versés au siège après un passage éclair de trois mois au parquet présentent énormément des difficultés pour instruire des dossiers devant le Tribunal et même afin de se prononcer sur la saisine du tribunal. Pour pallier, nous suggérons la tenue régulière des séminaires de renforcement de capacité pour que le système judiciaire devienne plus efficace.

Pour clore, nous proposons concrètement que conformément à la jurisprudence de 1998 qui a permis la révocation des magistrats ne remplissant pas les conditions de recrutement, le chef de l'Etat mette sur pied une commission pour analyser ce dossier et que tous ceux qui ont un diplôme autre que celui de Droit soient révoqués par le magistrat suprême. Lors du recrutement de 1998, tous ceux qui ne réunissaient pas les conditions d'admission furent révoqués par le chef de l'Etat.

En outre, le problème de corruption, clientélisme sont des maux qui rongent toute la société congolaise. Le système judiciaire étant le moteur de tout changement, il doit subir un travail de restructuration profonde afin d'influer sur toute la société congolaise. Et que le politique cesse de prendre le judiciaire en état. Nous suggérons la transformation de l'homme (magistrat) par une désintoxication visant un changement radical des mentalités. Bref, une légère augmentation du salaire des magistrats permettrait de réduire ce mal qu'est la corruption. Cela n'empêche que les magistrats corrompus fassent l'objet des poursuites et même encourir une radiation éventuelle si les faits s'avéraient vrais. 


\section{BIBLIOGRAPHIE}

\section{TEXTES JURIDIQUES.}

1. Loi organique $\mathrm{n}^{\circ} 06 / 020 \mathrm{du} 10$ octobre 2006 portant statut des magistrats.

2. Journal officiel du 25 octobre 2006.

3. Constitution du 18 février 2006.

4. Loi organique $n^{\circ} 08 / 013$ du 05 Août 2008 portant organisation et fonctionnement du Conseil Supérieur de la magistrature.

\section{OUVRAGES}

1. Guide pratique de procédure disciplinaire des magistrats, USAID, Pro justice, Médias Paul, Kinshasa, juillet 2011.

2. CMBACAU et SUR, S., Droit International Public, PUF, 7ème éd., Paris, 2006

3. RUBBENS, A., Le Droit Judiciaire Congolais, l'instruction criminelle et procédure pénale, T2 Mson F., Lancier, S.A., Bruxelles, 1965.

\section{ARTICLES ET AUTRES SOURCES}

1. KADIMA YVES, « Recrutement de nouveaux magistrats », in www.net,publié le 02 avril, 2011.

2. www.cd.undp.org

3. Décision $n^{\circ} 001 / \mathrm{CSM} / \mathrm{SPCSM} / \mathrm{D} / \mathrm{J} / 2010$ du 23 mars 2010 portant nomination de mille magistrats pour l'exercice 2010.

4. Décision $\mathrm{n}^{\circ} 002 / \mathrm{CSM} / \mathrm{SPCSM} / \mathrm{D} / \mathrm{J} / 2010$ du 23 mars 2010 portant réservation de mille autres magistrats pour l'an 2011. 